\title{
mRNA for N-Bak, a neuron-specific BH3-only splice isoform of Bak, escapes nonsense-mediated decay and is translationally repressed in the neurons
}

\author{
M Jakobson ${ }^{1}$, A Lintulahti ${ }^{1,2}$ and U Arumäe,
}

mRNA for neuronal Bak (N-Bak), a splice variant of pro-apoptotic Bcl-2 family member Bak is expressed in the neurons. Surprisingly the endogeneous N-Bak protein cannot be demonstrated in the neurons, although the antibodies recognize N-Bak protein from in vitro translation or transiently transfected cells. As N-Bak mRNA contains premature termination codon (PTC) at 89 nucleotides upstream from the last exon-exon junction, it could be degraded by nonsense-mediated decay (NMD) during the pioneer round of translation thus explaining the absence of the protein. We show here that the endogeneous neuronal N-Bak mRNA is not the NMD substrate, as it is not accumulating by cycloheximide treatment, it has a long lifetime, and even prevention of PTC by interfering with the alternative splicing did not lead to translation of the Bak mRNA. N-Bak protein is also not revealed by proteasome inhibitors. Our data suggest strong translational arrest of N-Bak mRNA in the neurons. We show that this arrest is partially mediated by $5^{\prime}$-untranslated region of Bak mRNA and it is not released during mitochondrial apoptosis.

Cell Death and Disease (2012) 3, e269; doi:10.1038/cddis.2012.4; published online 2 February 2012

Subject Category: Neuroscience

Nonsense-mediated mRNA decay (NMD) is a cellular surveillance mechanism to recognize and degrade mRNAs harboring premature translation-termination codon (PTC). Truncated proteins encoded by such mRNAs could be deleterious for the cell. During pioneer round of translation, the cell carries out quality control of the mRNA and either passes it to produce the proteins or directs it to the NMDmediated degradation. ${ }^{1,2}$ Specific multiprotein exon junction complexes deposited on the exon-exon junctions during premRNA splicing are detected and removed by the ribosome during pioneer round of translation. In mammals, one of the main criteria for the NMD substrate mRNAs is that distance of the PTC to the last (i.e. the $3^{\prime}$-most) exon-exon junction must be longer than 55 nucleotides ( 55 -nt NMD rule). ${ }^{3}$ If so, this last exon junction complex is recognized as a signal for NMD and the mRNA is degraded. ${ }^{2}$ Classically, the NMD is considered as a mechanism to remove aberrant mRNAs where PTC is generated by mutations. Many PTCs, however, are generated by physiological alternative splicing, where inclusion or exclusion of a cassette exon causes translational frameshift. About one-third of the alternatively spliced mRNAs is estimated to include isoforms with PTC that meet the 55-nt NMD rule and could potentially be degraded by NMD. ${ }^{4,5}$ To what extent such PTCs, generated by natural splicing process, actually trigger NMD is not clear.

We have described a splice variant of pro-apoptotic $\mathrm{BH} 1-3-$ domain Bcl-2 family member Bak that we named N-Bak for 'neuronal Bak' because it is expressed only in the neurons and not in any other tested primary cells or tissues, ${ }^{6}$ as also reported by others. ${ }^{7} \mathrm{~N}$-Bak is generated by inclusion of a 20 nucleotide cassette exon (exon $\mathrm{N}$ for 'neuronal') that causes a translational frameshift and PTC, such that instead of a BH1-3 Bak, a typical BH3-only protein N-Bak is encoded., ${ }^{6,8}$ The neurons splice Bak pre-mRNA completely into N-Bak transcripts such that the neurons specifically do not express Bak at all. ${ }^{6,8}$ Surprisingly we ${ }^{6}$ and others ${ }^{9}$ were not able to detect $\mathrm{N}$-Bak protein in the neurons (although Uo et al. ${ }^{8}$ reported the protein by immunoblot). The PTC on the N-Bak mRNA locates at 89 nucleotides upstream from the last exon-exon junction (Figure 1c) corresponding to the 55-nt NMD rule. Thus, the $\mathrm{N}$-Bak mRNA could be degraded during the pioneer round of translation, explaining the absence of the protein.

In this study we set up to test the hypothesis that N-Bak mRNA is degraded in the neurons by NMD that causes absence of the protein. Our results suggest that N-Bak mRNA is not the NMD substrate in the neurons and thus belongs to the mRNAs that escape the NMD despite the correspondence to the 55-nt NMD rule. Our data suggest that N-Bak mRNA is translationally arrested and its $5^{\prime}$-untranslated region (UTR) is partially responsible for this arrest.

\section{Results}

N-Bak mRNA but not protein is expressed in the neurons. Earlier we ${ }^{6}$ and others ${ }^{9}$ were not able to detect

${ }^{1}$ Institute of Biotechnology, University of Helsinki, FIN-00014 Helsinki, Finland

*Corresponding author: U Arumäe, Institute of Biotechnology, University of Helsinki, PO Box 56, Viikki Biocenter, FIN-00014 Helsinki, Finland. Tel: + 358919159396 ; Fax: + 3589 19159366; E-mail: urmas.arumae @ helsinki.fi

${ }^{2}$ Current address: Department of Dermatology and Allergology, University of Helsinki and Helsinki University Central Hospital, Helsinki, Finland.

Keywords: N-Bak; nonsense-mediated decay; alternative splicing; mRNA; cortical neurons

Abbreviations: NMD, nonsense-mediated decay; qRT-PCR, quantitative real-time PCR; PTC, premature termination codon; CHX, cycloheximide; ActD, actinomycin D; UTR, untranslated region

Received 16.8.11; revised 22.12.11; accepted 05.1.12; Edited by A Verkhratsky 
a

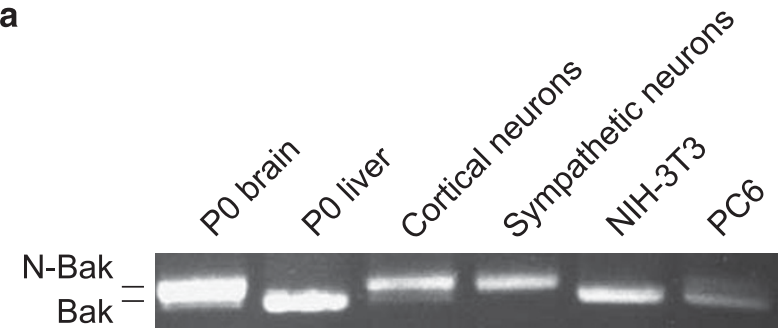

b

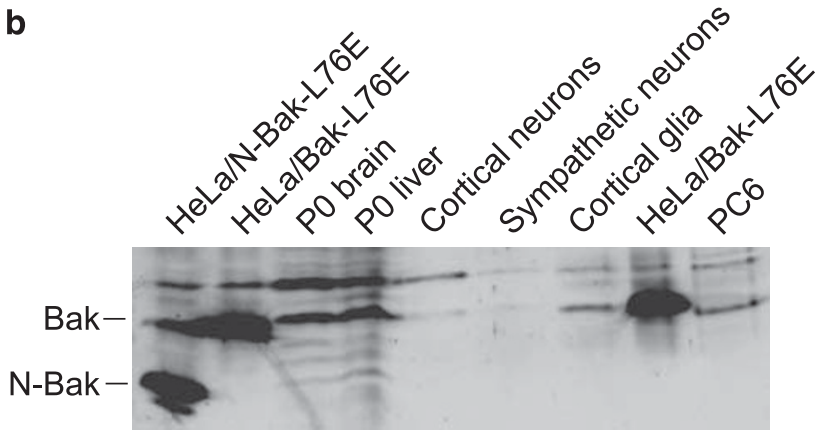

c

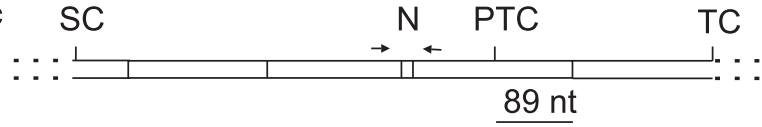

Figure 1 Expression of transcripts and proteins of Bak isoforms in the newborn (P0) mouse tissues, cultured mouse neurons, mouse NIH3T3 fibroblasts and rat pheochromocytoma PC6 cells. (a) RT-PCR detection of the transcripts of Bak $(510 \mathrm{nt})$ and N-Bak (530 nt). (b) Immunoblot from the other half of the same samples probed with anti-Bak antibodies (06-536, EMD Millipore). The positions of about $20 \mathrm{kD}$ N-Bak and about $25 \mathrm{kD}$ Bak proteins are shown on the lanes of HeLa cells overexpressing the respective apoptotically inactive L76E mutants. Note the absence of endogeneous N-Bak in all lanes. Pure glial cells from the P0 mouse cortex also expressed Bak but not N-Bak. Low levels of Bak in the cortical and sympathetic cultures come from the contaminating non-neuronal cells. Longer exposition of the filter still did not reveal N-Bak protein (not shown). (c) Schematic cartoon of the fragment of N-Bak mRNA showing start codon (SC), exon N (N), PTC and the authentic translation termination codon (TC). The exon-exon junctions are shown by vertical bars. Localization of the primers for PCR is shown by arrows

$\mathrm{N}$-Bak protein in the neurons. To study the issue further, we tested four anti-Bak antibodies: 06-536 (EMD Millipore/ Upstate, Darmstat, Germany), B-5897 (Sigma, St. Louis, MO, USA), Ab-2 (EMD Millipore/Calbiochem, Darmstat, Germany) and G-23 (Santa Cruz, sc-832, Heidelberg, Germany). First two antibodies are raised against the peptides from the $\mathrm{N}$-terminal region common for both Bak and N-Bak, whereas the epitopes for two latter antibodies are not precisely defined.

We first tested the antibodies on the in vitro translated $\mathrm{N}$-Bak and Bak and on the HeLa cells transiently transfected with the respective expression plasmids. Two antibodies (Sigma B-5897 and EMD Millipore 06-536) against the $\mathrm{N}$-terminal region of Bak/N-Bak recognized both about $25 \mathrm{kD}$ Bak and about $20 \mathrm{kD} \mathrm{N}$-Bak in either approach (Supplementary Figure 1) and these antibodies were used in the subsequent experiments. Other two antibodies (Ab-2 and G-23) did not show specific binding (data not shown). Antibody Ab-2 has been used ${ }^{8}$ to demonstrate N-Bak protein in the cortical neurons. Why it did not work in our hands is not clear. Immunostaining of the cultured neurons with any of the four anti-Bak antibodies results in high non-specific background (data not shown). Thus, at least two antibodies specifically detected N-Bak protein when it is expressed from the plasmid.

We then compared the levels of transcripts and proteins of Bak isoforms in the mouse brain. The cultured cells used in this study were analyzed as well. Transcripts for Bak isoforms were detected by RT-PCR with primers flanking exon $\mathrm{N}^{6}$ (Figure 1c), thus amplifying both mRNAs with comparable efficiency. As shown on Figure 1a, in the newborn (P0) mouse brain the level of $\mathrm{N}$-Bak transcripts (from the neurons) is highly surpassing the level of $\mathrm{BH} 1-3$ Bak transcripts (from the nonneuronal cells) that is in agreement with the results of RNase protection assay. ${ }^{6} \mathrm{~N}$-Bak transcripts were easily detected in the cultures of cortical and sympathetic neurons; the low levels of Bak transcripts in these cultures result from the few contaminating non-neuronal cells. ${ }^{6}$ Rat pheochromocytoma PC6 cells express exceptionally the mRNAs for both Bak isoforms, the levels of N-Bak mRNA being very low. As expected, only Bak but not N-Bak transcripts were detected in the PO mouse liver tissue and NIH-3T3 mouse fibroblast cells. The proteins were analyzed from the other half of the same samples by immunoblot. In agreement with previous study ${ }^{6}$ the anti-Bak antibody (EMD Millipore) did not detect N-Bak protein in any of the tissues or cells whereas the Bak protein was always detected (Figure 1b). The same results were obtained with antibody from Sigma, whereas other two antibodies gave again poor results (data not shown).

Thus, despite the high levels of N-Bak mRNA the protein was not detected in the neurons, although our assay easily revealed Bak protein from the samples with significantly lower Bak mRNA levels. We conclude that the absence of N-Bak protein in the neurons results from the posttranscriptional regulation rather than minute amounts of the mRNA. We considered three mechanisms that could lead to the absence of N-Bak protein: (i) rapid degradation of the mRNA, (ii) rapid degradation of the protein and (iii) translational block of the mRNA.

N-Bak mRNA is not the substrate for NMD. Inclusion of exon $\mathrm{N}$ to the Bak mRNA generates PTC that corresponds to the 55-nt NMD rule (Figure 1c). Such mRNA could potentially be degraded during the pioneer round of translation thus explaining the absence of $\mathrm{N}$-Bak protein. To test this hypothesis we blocked translation by cycloheximide ( $\mathrm{CHX}$ ) that should lead to rapid accumulation of the NMD substrate mRNAs. ${ }^{10,11}$ We treated cultured cortical neurons with $\mathrm{CHX}$ for 4 and $8 \mathrm{~h}$ and determined the levels of N-Bak transcripts by quantitative RT-PCR (qRT-PCR). mRNA for Bax, another pro-apoptotic Bcl-2 family member that does not contain PTC was also analyzed for comparison. The data were normalized to two reference mRNAs - neurofilament, medium polypeptide (NEFM) and enolase 2 (ENO2) that, just as $\mathrm{N}$ $\mathrm{Bak}$, are specifically expressed in the neurons but not in the small number of non-neuronal cells that are always present in the cultures. The fold changes were calculated in relation to samples treated with vehicle (DMSO) using relative expression software tool REST. ${ }^{12}$ As shown in Table 1, blockage of translation for $4 \mathrm{~h}$ did not lead to accumulation of $\mathrm{N}$-Bak or Bax transcripts in the cortical neurons, whereas 
Table 1 Relative changes of N-Bak and Bax transcripts in the cortical neurons treated with $\mathrm{CHX}$ or co-treated with cycloheximide (CHX) and actimomycin D (ActD) as determined by qRT-PCR and analyzed by relative expression software tool REST

\begin{tabular}{|c|c|c|c|c|c|c|c|}
\hline Transcript & Type & $\begin{array}{l}\text { Reaction } \\
\text { efficiency }\end{array}$ & Expression & S.E. & $95 \% \mathrm{Cl}$ & $\mathbf{P}(\mathrm{H} 1)$ & Result \\
\hline \multicolumn{8}{|c|}{$4 h \mathrm{CHX}(\mathrm{n}=3)$} \\
\hline Eno2 & REF & 0.9019 & 0.934 & & & & \\
\hline NEFM & REF & 0.8904 & 1.071 & & & & \\
\hline N-Bak & TRT & 0.8881 & 1.880 & $1.149-3.586$ & $0.828-4.237$ & 0.209 & \\
\hline Bax & TRT & 0.81 & 0.825 & $0.481-1.511$ & $0.341-2.241$ & 0.713 & \\
\hline \multicolumn{8}{|c|}{$8 h C H X(n=4)$} \\
\hline Eno2 & REF & 0.9019 & 0.981 & & & & \\
\hline NEFM & REF & 0.8904 & 1.020 & & & & \\
\hline N-Bak & TRT & 0.8881 & 2.890 & $2.229-3.846$ & $1.755-4.346$ & 0.020 & UP \\
\hline Bax & TRT & 0.81 & 1.076 & $0.599-1.720$ & $0.425-2.681$ & 0.821 & \\
\hline \multicolumn{8}{|c|}{$8 h C H X+A c t D(\mathrm{n}=4)$} \\
\hline Eno2 & REF & 0.8876 & 0.954 & & & & \\
\hline NEFM & REF & 0.8904 & 1.048 & & & & \\
\hline N-Bak & TRT & 0.9314 & 1.154 & $0.896-1.487$ & $0.768-1.806$ & 0.336 & \\
\hline Bax & TRT & 0.9589 & 1.046 & $0.508-2.055$ & $0.309-3.708$ & 0.891 & \\
\hline
\end{tabular}

Abbreviations: ENO2, enolase 2; NEFM, neurofilament, medium polypeptide. The data of the target transcripts (TRT) are normalized to the indicated reference transcripts $(\mathrm{REF})$. The sample groups $(\mathrm{CHX}$ or $\mathrm{CHX}+\mathrm{ActD})$ are compared with control groups $(\mathrm{DMSO})$. $\mathrm{P}(\mathrm{H} 1)$ designates the probability of alternate hypothesis that difference between sample and control groups is due only to chance $\mathrm{n}$ designates the number of independent repeat experiments

small but significant accumulation of $\mathrm{N}$-Bak but not Bax occurs by $8 \mathrm{~h}$ of $\mathrm{CHX}$ treatment. However, this increase was completely blocked by transcription inhibitor actinomycin $\mathrm{D}$ (ActD). Thus, this late and small increase in the N-Bak mRNA was caused by increased transcription, most probably because of $\mathrm{CHX}$-induced cellular stress. ${ }^{13}$ The same results were obtained with another pair of $\mathrm{N}$-Bak-specific primers (mouse N-Bak-2, data not shown), or when data were calculated in relation to untreated samples instead of DMSOtreated samples (data not shown). In the PC6 cells, similar $\mathrm{CHX}$-induced increase in the N-Bak mRNA level (normalized to beta actin) was observed by $4 \mathrm{~h}$ of treatment but was also completely blocked by co-treatment with ActD (data not shown).

As a rule, the NMD substrate mRNA levels are low because of ongoing degradation. ${ }^{14,15}$ If N-Bak mRNA were a substrate for NMD in the neurons, its turnover should be extremely rapid, as the levels of this transcript in the brain and cultured neurons are high (Figure 1). To study the stability of N-Bak mRNA we blocked transcription in the cortical cultures by ActD and determined the time-dependency of N-Bak and Bax mRNA degradation by qRT-PCR. The data were normalized to 7SL RNA (RN7SL1) and 18s ribosomal RNA (Rn18s) that are transcribed by RNA polymerase III and thus not blocked by ActD. As shown on Figure 2, the N-Bak transcripts were stabile in the cortical neurons, whereas the levels of Bax transcripts decreased in time (Figure $2 b$ ). Longer treatments with ActD caused general deterioration of the cortical cultures that makes the analysis unreliable. Similar results were obtained using another pair of N-Bak-specific primers (mouse N-Bak-2, data not shown). In the ActD-treated PC6 cells, the transcripts of $\mathrm{N}$-Bak decreased with the same rate as the transcripts of Bax (data not shown).

In summary, the N-Bak mRNA is remarkably stabile in the neurons. Such stability is not compatible with the NMD substrate transcripts. Thus, our data do not support the hypothesis that $\mathrm{N}-\mathrm{Bak}$ mRNA is degraded in the neurons by NMD.

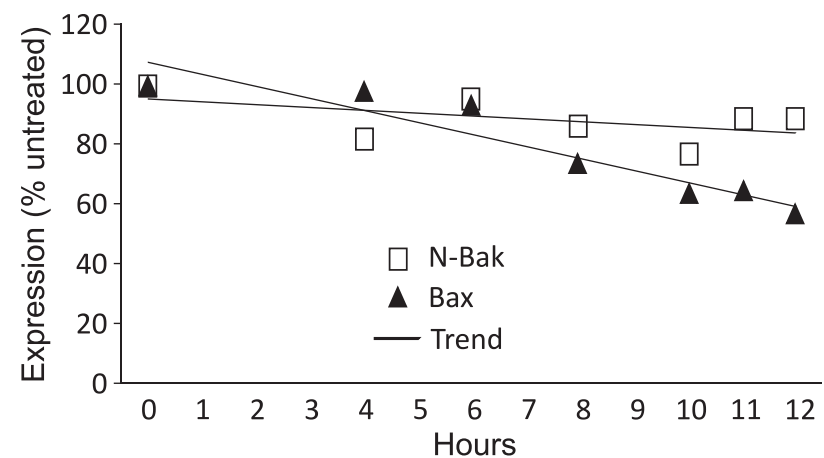

Figure 2 Stability of N-Bak mRNA. Cortical neurons were treated with the transcription inhibitor ActD $(5 \mu \mathrm{g} / \mathrm{ml})$ and analyzed by qRT-PCR at indicated time points. The levels of N-Bak and Bax transcripts were normalized to 7SL RNA (RN7SL1) and Rn18s. Each time point was calculated in relation to untreated samples by relative expression software tool REST. The trendlines are shown by black lines. The data are results of three independent experiments

Prevention of alternative splicing and PTC did not lead to translation of N-Bak mRNA. The experiments with $\mathrm{CHX}$ and ActD treatment described above suggest that despite of the PTC falling under the 55-nt NMD rule, N-Bak mRNA is not the NMD substrate in the neurons. To study the issue further we tested whether this mRNA could be translated without PTC. To that end we designed Vivo-Morpholino oligonucleotides against exon $\mathrm{N}$ that should prevent its inclusion into the endogeneous mRNA. Without exon $\mathrm{N}$ the mRNA has no PTC and encodes for the BH1-3 Bak in the neurons. Application of the exon $\mathrm{N}$-specific oligonucleotides to the culture medium of cortical neurons for $24 \mathrm{~h}$ indeed leads to significant appearance of Bak mRNA with concomitant disappearance of N-Bak mRNA, whereas control oligonucleotides had no effect (Figure 3a). However, immunoblot with anti-Bak antibody (06-536, EMD Millipore) did not reveal upregulation of Bak protein in such cortical cultures forced to express Bak mRNA (Figure 3b). Addition of 
a

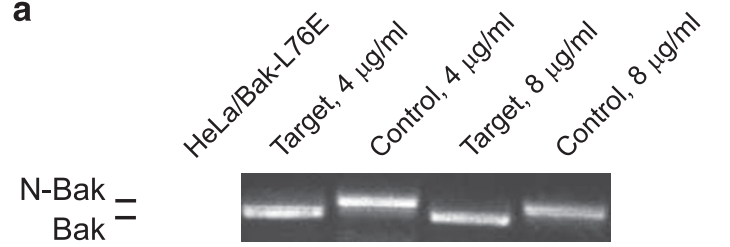

b

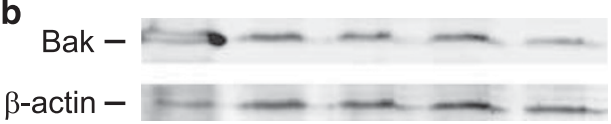

Figure 3 Bak mRNA is not translated in the absence of PTC. (a) RT-PCR analysis of the transcripts of Bak (510 nt) and N-Bak (530 nt) in the cultured cortical neurons treated with exon $\mathrm{N}$-targeting or control Vivo-Morpholino oligonucleotides at 4 or $8 \mu \mathrm{g} / \mathrm{ml}$ for $24 \mathrm{~h}$. N-Bak mRNA is converted into Bak mRNA with exon $\mathrm{N}$-specific (target oligos) but not control oligos. Qualitatively same results were obtained in three independent experiments. (b) Upper panel: immunoblot with Bak-specific antibodies (06-536, EMD Millipore) from the other half of the same samples as in (a). The position of about 25-kD Bak is shown on the lane of HeLa cells overexpressing Bak-L76E. The Bak on the neuronal lanes comes from the contaminating glial cells and its level is not increased by the oligonucleotide treatment. Lower panel: reprobing the same filter with antibodies to $\beta$-actin. Qualitatively same results were obtained in three independent experiments

caspase inhibitor BAF or proteasome inhibitor MG-132 to the culture medium still did not lead to increase in the Bak protein levels in the oligonucleotide-treated cultures (data not shown). Thus, despite the absence of exon N and PTC the endogenous Bak mRNA was not translated in the neurons. The data suggest that a translational arrest rather than NMD causes the absence of $\mathrm{N}-\mathrm{Bak}$ protein.

$\mathrm{N}$-Bak protein is not rapidly degraded by proteasome. One explanation for the absence of N-Bak protein could be its rapid proteasomal degradation. In support of this possibility, the Mobyle program predicted a PEST sequence (signal for proteasome-mediated degradation) encompassing residues 12-33 of mouse Bak/ $\mathrm{N}$-Bak. To address this alternative we treated the cortical neurons with proteasome inhibitor MG-132 in the presence or absence of caspase inhibitor BAF. N-Bak protein was not detectable in these conditions whereas control heat shock protein (Hsp70) was strongly upregulated by proteasome inhibition (Figure 4), as published. ${ }^{16}$ Thus we did not find evidences for rapid proteasome-mediated degradation of $\mathrm{N}-\mathrm{Bak}$ protein. Moreover, the BH1-3 Bak protein is easily detected in many cells and tissues despite the presence of this predicted PEST sequence.

$\mathrm{N}$-Bak protein was not induced in the apoptotic neurons. As a typical BH3-only protein, N-Bak could function in the Bax-dependent mitochondrial apoptosis. If so, the translational arrest could be released and N-Bak protein expressed in the apoptotic neurons. We treated the cortical neurons with etoposide, the inhibitor of topomerase II that triggers mitochondrial apoptosis, for 24-36 h. The death of the neurons was blocked by caspase inhibitor BAF with or without proteasome inhibitor. Immunoblot with anti-Bak antibodies (06-536, EMD Millipore) (Figure 5) or Sigma (data not shown) again did not show appearance of N-Bak protein in any of these conditions. The same result was
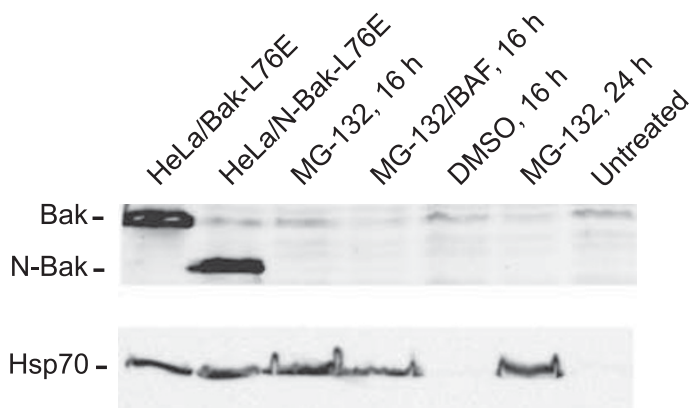

Figure 4 N-Bak protein is not revealed by proteasome inhibition. Cortical neurons were treated with proteasome inhibitor MG-132 for 16 or $24 \mathrm{~h}$ in the presence or absence of caspase inhibitor BAF, or with the vehicle (DMSO). Upper panel shows the immunoblot with anti-Bak antibodies (06-536, EMD Millipore). Shown are the samples from HeLa cells transiently transfected with the inactive L76E mutants of Bak and N-Bak. Lower panel shows the same filter reprobed with anti-Hsp70 antibodies. Proteasome inhibition did not reveal N-Bak protein in the cortical neurons, although the positive control Hsp70 was strongly induced. The HeLa cells express Hsp70 constitutively. The same results were obtained in three independent experiments

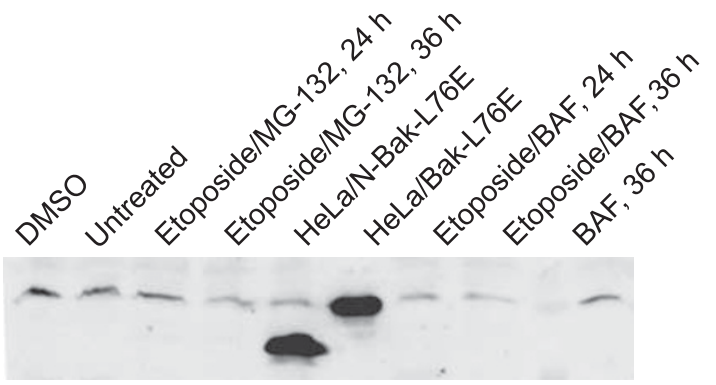

Figure $5 \mathrm{~N}$-Bak protein is not induced in the apoptotic neurons. Cortical neurons were treated with etoposide for 24 or $36 \mathrm{~h}$ in the presence of proteasome inhibitor MG-132 or caspase inhibitor BAF. Untreated and vehicle (DMSO)-treated cultures as well as HeLa cells overexpressing apoptotically inactive L76E mutants of $\mathrm{N}$-Bak and Bak were included as controls. The immunoblot is probed with anti-Bak antibody (06-536, EMD Millipore). The same results were obtained in three independent experiments

obtained when the sympathetic neurons were deprived of nerve growth factor, or treated with etoposide for three days in the presence of BAF (data not shown). Thus, N-Bak mRNA is not translated during the mitochondrial apoptosis, at least not at the detectable level. The membranes were reprobed with antibody Ab-2 used by others to demonstrate $\mathrm{N}$-Bak in the camptothecin-treated cortical cultures ${ }^{8}$ but no specific binding was detected (data not shown).

Translational arrest of N-Bak mRNA is partially mediated by $5^{\prime}$-UTR. To study the mechanism of translational arrest of N-Bak mRNA we focused on its $5^{\prime}$-UTR that contains two open reading frames upstream of the translation initiation codon. Such $5^{\prime}$-UTRs can participate in the translational repression of mRNAs. ${ }^{17,18}$ We tested the effect of Bak $5^{\prime}$-UTR on the translation using Dual Luciferase Reporter assay with Firefly and Renilla luciferase (FF-Luc and Rn-Luc, respectively). The $5^{\prime}$-UTR of mouse Bak mRNA was cloned upstream of the Firefly luciferase reporter gene, resulting in a Bak-5'-UTR-FF-Luc construct, whereas the reporter without the Bak 5'-UTR (FF-Luc) was used for a reference. 
Sympathetic neurons were microinjected with the plasmid mixtures of either Bak-5'-UTR-FF-Luc/Rn-Luc or FF-Luc/ Rn-Luc. Relative luciferase activity was determined $48 \mathrm{~h}$ later by normalization of FF-Luc activity to Rn-Luc activity. The activity of luciferase in the Bak-5'-UTR-FF-Luc-injected neurons constituted $37.6 \pm 3.6 \%$ (S.E.M., $n=3$ ) of that in the FF-Luc-injected neurons after $48 \mathrm{~h}$. Thus, the presence of Bak $5^{\prime}-$ UTR in front of the FF-Luc gene strongly reduced its activity.

\section{Discussion}

The main aim of this study was to address whether the N-Bak mRNA is the substrate for NMD that could explain the absence of the encoded protein in the neurons. Inclusion of a 20-nucleotide exon $\mathrm{N}$ to $\mathrm{N}$-Bak mRNA causes a translational frameshift and PTC that corresponds to the 55-nt rule of NMD. Such mRNAs should be degraded in the pioneer round of translation and could therefore not produce significant amount of the protein.

The main finding of this study is that N-Bak mRNA is not the substrate for NMD in the neurons. The evidences for this conclusion are the following. (i) Blockage of translation with $\mathrm{CHX}$ did not lead to rapid and massive accumulation of $\mathrm{N}-\mathrm{Bak}$ mRNA in the cortical neurons. (ii) mRNA for N-Bak was remarkably stable in the neurons that is not compatible with translation-associated rapid degradation. (iii) Abrogation of PTC from the neuronal N-Bak mRNA did not lead to its translation. We conclude that neurons have specific mechanism(s) to avoid NMD of the N-Bak mRNA, possibly via translational repression. Of note, we studied here the endogenous PTC-containing transcripts in their natural neuronal environment instead of tumor cell lines transfected with artificial plasmid constructs that might not be subjected to all cellular regulations. On the other hand, blockage of endogeneous NMD by siRNAs to Upf1/RENT ${ }^{15}$ that is a common approach to identify NMD substrates appeared technically too complicated in the primary neurons.

Endogeneous $\mathrm{N}-\mathrm{Bak}$ protein has been detected by others by antibody $\mathrm{Ab}-2 .^{8}$ We put special efforts to repeat these results but in our hands the antibody was not specific, even in the HeLa cells transfected with inactive Bak. The reason for this discrepancy is not clear and formally it cannot be excluded that we have not found the proper conditions for N-Bak immunoblot. However, different biochemical approaches including various solubilization buffers, immunoprecipitations and so on never revealed endogenous $\mathrm{N}$-Bak protein with any anti-Bak antibody tested, although plasmid-expressed N-Bak was always seen. ${ }^{6}$

Classically, the NMD has been described for mRNAs where the PTC is generated by aberrations. However, it is estimated that about one-third of alternatively spliced mRNAs have PTC-containing isoform(s) that could potentially be degraded by NMD. ${ }^{4,5}$ Such physiological generation of PTC could be a mechanism to regulate gene expression, a process termed regulated unproductive splicing and translation (RUST). ${ }^{19}$ Although the issue is not clear, some studies estimated that only up to $10 \%$ of such transcripts accumulate when NMD is blocked $^{15,20,21}$ showing that a considerable portion can escape the NMD. Our data suggest that N-Bak mRNA is not the typical case of RUST, where the alternative splicinggenerated PTC is exploited to downregulate the mRNA. ${ }^{22-24}$ Instead, the mRNA appears to be maintained in a translationally repressed state independently (and despite) of the PTC (Figure 3). N-Bak mRNA thus belongs to the class of NMD-escape mRNAs which are translationally repressed. ${ }^{25}$

The mechanisms of translational arrest and the strong stability of N-Bak mRNA remain to be studied. Our results show that the $5^{\prime}$-UTR of Bak mRNA strongly reduces the translation of the reporter gene in the neurons, suggesting that it participates in the translational repression of N-Bak mRNA. $5^{\prime}$-UTR of mouse, rat and human Bak contain short open reading frames upstream of the translation initiation site shown to be essential in the translational repression of the mRNAs. ${ }^{17,18}$ However, the 5'UTR of Bak did not repress expression of the reporter gene completely, suggesting the participation of other mechanism as well. The potential candidates are the microRNAs known to repress mRNA translation via $3^{\prime}-\mathrm{UTR}^{26-29}$

The splicing of Bak pre-mRNA (inclusion of exon $N$ ) is triggered around the time the neuronal progenitors exit the cell cycle (our unpublished data). Moreover, the sequence, regulatory elements (branch point and poly-pyrimidine tract) and the position of exon $\mathrm{N}$ between constitutive exons 4 and 5 of the Bak gene are evolutionally conserved. Thus, the absence of Bak (and presence of N-Bak mRNA) is a panneuronal feature that seems to last throughout the lifetime of the neurons. It is tempting to speculate that the biological meaning of neuronal Bak pre-mRNA splicing is to get rid of an apoptotic effector Bak. Indeed, the death machinery is under stringent brakes in the postmitotic neurons, such as blockage of Bax protein, ${ }^{30}$ strong association of the caspases with XIAP protein, ${ }^{31-33}$ low levels of Apaf-1 (Wright et al. ${ }^{34}$, Wright et al. $^{35}$ ) and so on. Removal of Bak by alternative splicing could be one additional brake to make the neurons more resistant to accidental apoptosis. The biological meaning of $\mathrm{N}$-Bak mRNA remains currently unclear. Exon $\mathrm{N}$ is inserted to the Bak pre-mRNA with exact precision (just before the $\mathrm{BH} 1$ domain-encoding sequence) that converts the $\mathrm{BH} 1$ 3-type protein into the BH3-only protein. Thus, the neurons seem to require an additional member to their repertoire of $\mathrm{BH} 3-$ only proteins and generate it by alternative splicing of Bak. Curiously, the N-Bak mRNA is stabilized and translationally repressed in the neurons, and this repression is not relieved in the classical apoptotic situations (this study) or in many other stress conditions (our unpublished data). When (if ever) this mRNA is translated remains currently unknown.

\section{Materials and Methods}

Cell cultures. The cortical neurons from the embryonic day 15-16 mice, dissociated as published, ${ }^{6}$ were grown on the polyornithine-coated $35-\mathrm{mm}$ plastic dishes (Becton Dickinson, Franklin Lakes, NJ, USA) in the Neurobasal medium (Invitrogen, Carlsbad, CA, USA) containing $2 \%$ of B27 serum supplement (Invitrogen), $0.5 \mu \mathrm{M}$ L-Glutamine and $100 \mu \mathrm{g} / \mathrm{ml}$ Primocin (InvivoGen, San Diego, CA, USA) for 5- 6 days before the experiments. The cultures containing many nonneuronal cells were excluded, although completely glia-free cultures were not possible. The experiments were repeated on the independent cultures 3-5 times. The newborn mouse sympathetic neurons were cultured on the dishes coated with polyornithine-laminin (Sigma) in the Neurobasal medium containing B27 supplement (Invitrogen) and $30 \mathrm{ng} / \mathrm{ml}$ mouse 2.5 S NGF (Promega, Madison, WI, USA), as published. ${ }^{36,37}$ PC6 cells were grown in DMEM (Invitrogen) containing 
$10 \%$ of horse serum (PAA Laboratories, Pasching, Austria), $5 \%$ of fetal bovine serum (HyClone, Thermo Scientific, Loughborough, UK) and $100 \mu \mathrm{g} / \mathrm{ml}$ of Normocin (InvivoGen); HeLa, primary glia and NIH-3T3 cells were grown in DMEM (Invitrogen) containing $10 \%$ fetal bovine serum (HyClone) and $100 \mu \mathrm{g} / \mathrm{ml}$ Normocin. The following additives were used: CHX (Sigma) in DMSO at $20 \mu \mathrm{g} / \mathrm{ml}$, ActD (Sigma) at $5 \mu \mathrm{g} / \mathrm{ml}$, proteasome inhibitor MG-132 (Sigma) at $10 \mu \mathrm{g} / \mathrm{ml}$, pan-caspase inhibitor boc-aspartyl(OMe)-fluoromethylketone (BAF) (Calbiochem/EMD Biosciences, Darmstadt, Germany) at $25 \mu \mathrm{g} / \mathrm{ml}$, etoposide (Sigma) at $4 \mu \mathrm{g} / \mathrm{ml}$. The cell membrane-penetrating Vivo-Morpholino oligonucleotides (GeneTools, LLC, Philomath, OR, USA) were designed against the region encompassing exon $\mathrm{N}$ (5'-ACCTGTGCATGTTGCTGCTGGCCT-3'), control sequence (5'-AGCGCACGA TCAACATGCAGACGG-3') and applied to the culture medium at 4 or $8 \mu \mathrm{g} / \mathrm{ml}$ for 8 or $24 \mathrm{~h}$.

Primers. PCR primers were designed using Primer Express v2.0. software (Applied Biosystems, Carlsbad, CA, USA) or OligoAnalyzer software (eu.idtdna.com) and ordered from Sigma. PCR primers:

N-Bak/Bak F: 5'-TTGCCCAGGACACAGAGGAGGT-3', N-Bak/Bak R: 5'-GAA TTGGCCCAACAGAACCACACC- $3^{\prime} .6$ mBak 5'-UTR F: $5^{\prime}$-GATTAAGCTTGGAGCT GGGACCTCCTCTATG-3', mBak 5'-UTR R: 5'-ACTTAA GCTTGAATTCACCACC GTCACTTGTCAC-3'.

Quantitative PCR primers: mouse 7SL ncRNA F: $5^{\prime}$-CGATCGGGTGTCC GCACTAAGT-3' R: $5^{\prime}$-TGGTCGTTCACCCCTCCTTAGG-3', rat 7SL ncRNA F: 5'-GTTTCCGACCTGCGCCGGTTC-3' R: 5'-TATGCCGATCGGGTGTCCGCAC-3', Rn18s F: 5'-GCACGGCCGGTACAGTGAAACT-3', R: 5'-AGCGCCCGTCGGCAT GTATTAG-3', mouse N-Bak 1 F: 5'-TGCTGATGGCAACTTCAACTG-3' R: 5 $^{\prime}$-AG CCAAAGCCCAGGAGAG-3', mouse N-Bak 2 F: $5^{\prime}$-CAGCAACATGCACAGCCT AT-3' R: 5'-CTGTGCATGTTGCTGCTGG-3', Bax F: 5'-TTGCTGATCTTGTCGT CGGACTGT-3' R: $5^{\prime}$-CAGCCCATGATGGTTCTGATC-3', ENO2 F: 5' ${ }^{\prime}$-CTGCAGT CCAAGAGCATCGA-3' R: 5'-ATGAGCTGGTTGTACTTCGCCAGA-3', NEF-M F: 5'-GCTACGACACGGAGTTCCAGA-3' R: 5'-ACTGCTGGATGGTGTCCTGG-3', rat N-Bak F: 5'-ATGGAATCCTGTGGCATCCAT-3' R: 5'-GTGCATGCCGCTGCT GCTG-3', Actb F: 5'-ATGGAATCCTGTGGCATCCAT-3' R: 5'-CCACCAGACAAC ACTGTGTTGG-3'.

Cloning. Mouse BAK1 $5^{\prime}$-UTR fragment (bp -247-19) was amplified by PCR from the whole E10.5. embryo cDNA using primers mBak $5^{\prime}$-UTR $F$ and mBak $5^{\prime}$-UTR R. Purified PCR product was inserted into the Hindlll site of $p G L 4.13$ vector (Promega) resulting in Bak-5'-UTR-FF-Luc-pGL4.13 construct, named Bak-5'-UTRFF-Luc. The expression plasmids for N-Bak, Bak, N-Bak-L76E and Bcl-xL are published. ${ }^{6,36,38}$

Microinjections and luciferase assay. Microinjection of the neurons was performed essentially as published. ${ }^{6,36}$ Briefly, the neurons were grown with NGF for 5-6 days and pressure-microinjected with the expression plasmids: either Bak-5'-UTR-FF-Luc-pGL4.13 or FF-Luc-pGL4.13 $(10 \mathrm{ng} / \mu$ l), both mixed with Rn-Luc-pGL4.73 $(5 \mathrm{ng} / \mu \mathrm{l})$. In all 200-300 neurons were injected per experimental point. Forty-eight hours later the neurons were lysed in the Passive Lysis Buffer and the luciferase activities determined using Dual-Luciferase Reporter Assay System (Promega) and GloMax 20/20 luminometer (Promega) according to the manufacturer's instructions. The experiments were repeated three times on independent cultures.

RT-PCR. Total RNA was extracted from the P0 mouse brain and liver, from whole mouse embryos (E10.5) or from cultured cells by TRI-reagent (Molecular Research Center, Cincinnati, OH, USA) according to the manufacturer's instructions. Isolated RNA was dissolved in the RNase-free water and treated with DNAse I and removal reagents from TURBO DNA-free kit (Ambion, Austin, TX, USA). Total RNA concentrations and absorbance ratios were measured by NanoDrop spectrophotometer. In all, $2 \mu \mathrm{g}$ of isolated RNA was used for cDNA synthesis in the $20 \mu \mathrm{l}$ reaction volume using Transcriptor First Strand cDNA Synthesis kit (Roche Diagnostics, Mannheim, Germany). Random hexamer primers were used for CDNA priming. One out of ten of the CDNA mixture was used in PCR with Phusion High Fidelity DNA Polymerase (Finnzymes, Vantaa, Finland). PCR conditions are published previously. ${ }^{6}$

qRT-PCR. qPCR reactions were performed in triplicates using LightCycler detection system LC480 (Roche Diagnostics). Shortly, $5 \mu$ l of $1: 10$ diluted cDNA was used as a template in a mixture of specific pair of primers and LightCycler 480
SYBR Green I Master kit (Roche). The amplification cycle was repeated 45 times with all samples as follow: $95^{\circ} \mathrm{C}$ for $10 \mathrm{~s}$ with temperature transition rate $4.4^{\circ} \mathrm{C} / \mathrm{s}$, $60^{\circ} \mathrm{C}$ for $15 \mathrm{~s}$ with temperature transition rate $2.2^{\circ} \mathrm{C} / \mathrm{s}$ and $72{ }^{\circ} \mathrm{C}$ for $20 \mathrm{~s}$ with temperature transition rate $4.4^{\circ} \mathrm{C} / \mathrm{s}$. The changes in the mRNA levels following the treatments were analyzed by relative expression software tool REST. ${ }^{12}$ The MIQE guidelines ${ }^{39}$ were followed in assay design and analysis. The levels of the reference transcripts did not change significantly by $\mathrm{CHX}$ treatment, as validated by the $2^{-\mathrm{CT}}$ method, ${ }^{40} P$-values were calculated by Student's $t$-test: fold change of treated samples compared with DMSO-treated samples was 1.2 for ENO2 $(P=0.6), 1.5$ for NEFM $(P=0.5)$ and 1.1 for Actb $(P=0.4)$. As expected, ActD treatment did not change the levels of RNA polymerase III-transcribed reference genes, calculated at $8 \mathrm{~h}$ treatment: fold change of treated samples compared with non-treated samples for 7SLRNA was $0.95(P=0.4)$ in the cortical neurons and $0.92(P=0.4)$ in the PC6 cells. For Rn18s: $0.7(P=0.3)$ in the cortical neurons and $0.93(P=0.3)$ in the PC6 cells.

Immunoblots. Brain and liver samples for immunoblotting were homogenized in the extraction buffer containing $0.3 \mathrm{M}$ sucrose, $10 \mathrm{mM}$ Hepes, pH 7.2, $1 \mathrm{mM}$ EDTA and EDTA-free protease inhibitor cocktail (CompleteMini, Roche) and then $5 \times$ Laemmli sample buffer was added. Cultured cells were lysed in $2.5 \times$ SDS sample buffer ( $160 \mu / / 35 \mathrm{~mm}$-dish). Samples were analyzed by SDS-PAGE in the $15 \%$ gels, blotted to nitrocellulose membranes and blocked with $5 \%$ non-fat dry milk in TBS containing $0.1 \%$ Tween-20 (TBST). Membranes were probed with the anti-Bak antibodies from EMD Millipore (06-536), Sigma (B-5897), Santa Cruz Biotechnology, Inc., (G-23, sc-832), or Ab-2 (TC-102) (EMD Millipore, AM04). Other antibodies were anti-Hsp70 from Stressgen Biotechnologies, Victoria, BC, Canada (SPA810), anti- $\beta$-actin (Sigma) and anti- $\alpha$-tubulin (Sigma). The blots were then probed with the secondary antibodies: goat anti-mouse from DAKO (Golstrup, Dennmark) or donkey anti-rabbit from GE Healthcare (Buckinghamshire, UK). Immunoblots were developed using SuperSignal West Pico Chemiluminecent Substrate (Pierce Biotechnology, Rockford, IL, USA) and Fujifilm LAS3000 imager (Fuji Photo Film, Tokyo, Japan). For re-probing the filters were stripped with washing buffer: $62.5 \mathrm{mM}$ Tris- $\mathrm{HCl}$, pH 6.8 containing $2 \%$ SDS and $100 \mathrm{mM}$ 2-mercaptoethanol for $15 \mathrm{~min}$ at $+55^{\circ} \mathrm{C}$ and washed further with PBS and TBST.

Biochemical methods. In vitro translation was performed in the rabbit reticulocyte lysate using TnT Quick Coupled Transcription/Translation System (Promega) in the presence of ${ }^{35} \mathrm{~S}$-methionine (Perkin-Elmer, Waltham, MA, USA) according to the manufacturer's instructions. HeLa cells were transfected by Lipofectamine 2000 (Invitrogen). Blockage of translation by $\mathrm{CHX}$ was optimized by metabolic labeling of SCG neurons followed by precipitation of the proteins by trichloro-acetic acid and measuring the radioactivity by scintillation beta-counter. Treatment with $20 \mu \mathrm{g} / \mathrm{ml}$ of $\mathrm{CHX}$ for $4 \mathrm{~h}$ blocked $90-95 \%$ of new protein translation.

\section{Conflict of Interest}

The authors declare no conflict of interest.

Acknowledgements. This study was financed from the Academy of Finland program 11186236 (Finnish Centre of Excellence Program 2008-2013) and the Academy of Finland research fellow grant for U.A. We thank Congjun Zheng and Zeren Basaran for excellent technical assistance. Mikko Frilander is acknowledged for invaluable advices. We are indebted to Madis Jakobson and Anmol Kumar for discussions and shared reagents.

1. Silva AL, Romao $L$. The mammalian nonsense-mediated mRNA decay pathway: to decay or not to decay! Which players make the decision? FEBS Lett 2009; 583: 499-505.

2. Reznik $B$, Lykke-Andersen J. Regulated and quality-control mRNA turnover pathways in eukaryotes. Biochem Soc Trans 2010; 38: 1506-1510.

3. Nagy E, Maquat LE. A rule for termination-codon position within intron-containing genes: when nonsense affects RNA abundance. Trends Biochem Sci 1998; 23: 198-199.

4. Lewis BP, Green RE, Brenner SE. Evidence for the widespread coupling of alternative splicing and nonsense-mediated mRNA decay in humans. Proc Natl Acad Sci USA 2003; 100: 189-192.

5. Green RE, Lewis BP, Hillman RT, Blanchette M, Lareau LF, Garnett AT et al. Widespread predicted nonsense-mediated mRNA decay of alternatively-spliced transcripts of human normal and disease genes. Bioinformatics 2003; 19 (Suppl 1): i118-i121.

6. Sun YF, Yu LY, Saarma M, Timmusk T, Arumae U. Neuron-specific Bcl-2 homology 3 domain-only splice variant of Bak is anti-apoptotic in neurons, but pro-apoptotic in non-neuronal cells. J Biol Chem 2001; 276: 16240-16247. 
7. Wong HK, Fricker M, Wyttenbach A, Villunger A, Michalak EM, Strasser A et al. Mutually exclusive subsets of $\mathrm{BH} 3$-only proteins are activated by the p53 and c-Jun $\mathrm{N}$-termina kinase/c-Jun signaling pathways during cortical neuron apoptosis induced by arsenite. Mol Cell Biol 2005; 25: 8732-8747.

8. Uo T, Kinoshita Y, Morrison RS. Neurons exclusively express N-Bak, a BH3 domain-only Bak isoform that promotes neuronal apoptosis. J Biol Chem 2005; 280: 9065-9073.

9. Putcha GV, Harris CA, Moulder KL, Easton RM, Thompson CB, Johnson Jr EM. Intrinsic and extrinsic pathway signaling during neuronal apoptosis: lessons from the analysis of mutant mice. J Cell Biol 2002; 157: 441-453.

10. Belgrader $\mathrm{P}$, Cheng J, Maquat LE. Evidence to implicate translation by ribosomes in the mechanism by which nonsense codons reduce the nuclear level of human triosephosphate isomerase mRNA. Proc Natl Acad Sci USA 1993; 90: 482-486.

11. Boutz PL, Stoilov P, Li Q, Lin CH, Chawla G, Ostrow K et al. A post-transcriptiona regulatory switch in polypyrimidine tract-binding proteins reprograms alternative splicing in developing neurons. Genes Dev 2007; 21: 1636-1652.

12. Pfaffl MW, Horgan GW, Dempfle L. Relative expression software tool (REST) for group wise comparison and statistical analysis of relative expression results in real-time PCR. Nucleic Acids Res 2002; 30: e36.

13. Ionov Y, Nowak N, Perucho M, Markowitz S, Cowell JK. Manipulation of nonsense mediated decay identifies gene mutations in colon cancer Cells with microsatellite instability. Oncogene 2004; 23: 639-645.

14. Losson R, Lacroute $F$. Interference of nonsense mutations with eukaryotic messenger RNA stability. Proc Natl Acad Sci USA 1979; 76: 5134-5137.

15. Pan Q, Saltzman AL, Kim YK, Misquitta C, Shai O, Maquat LE et al. Quantitative microarray profiling provides evidence against widespread coupling of alternative splicing with nonsense-mediated mRNA decay to control gene expression. Genes Dev 2006; 20 153-158.

16. Wang $X$, Pongrac JL, DeFranco DB. Glucocorticoid receptors in hippocampal neurons that do not engage proteasomes escape from hormone-dependent down-regulation but maintain transactivation activity. Mol Endocrinol 2002; 16: 1987-1998.

17. Ajay SS, Athey BD, Lee I. Unified translation repression mechanism for microRNAs and upstream AUGs. BMC Genomics 2010; 11: 155.

18. lacono M, Mignone F, Pesole G. UAUG and UORFs in human and rodent $5^{\prime}$ untranslated mRNAs. Gene 2005; 349: 97-105

19. Lareau LF, Brooks AN, Soergel DA, Meng Q, Brenner SE. The coupling of alternative splicing and nonsense-mediated mRNA decay. Adv Exp Med Biol 2007; 623: 190-211.

20. Mendell JT, Sharifi NA, Meyers JL, Martinez-Murillo F, Dietz HC. Nonsense surveillance regulates expression of diverse classes of mammalian transcripts and mutes genomic noise. Nat Genet 2004; 36: 1073-1078.

21. Mcllwain DR, Pan Q, Reilly PT, Elia AJ, McCracken S, Wakeham AC et al. Smg1 is required for embryogenesis and regulates diverse genes via alternative splicing coupled to nonsense-mediated mRNA decay. Proc Natl Acad Sci USA 2010; 107: 12186-12191.

22. Zhang Z, Zhou L, Hu L, Zhu Y, Xu H, Liu Y et al. Nonsense-mediated decay targets have multiple sequence-related features that can inhibit translation. Mol Syst Biol 2010; 6: 442

23. Gardner LB. Nonsense-mediated RNA decay regulation by cellular stress: implications for tumorigenesis. Mol Cancer Res 2010; 8: 295-308.

24. McGlincy NJ, Smith CW. Alternative splicing resulting in nonsense-mediated mRNA decay: what is the meaning of nonsense? Trends Biochem Sci 2008; 33: 385-393.
25. You KT, Li LS, Kim NG, Kang HJ, Koh KH, Chwae YJ et al. Selective translational repression of truncated proteins from frameshift mutation-derived mRNAs in tumors. PLOS Biol 2007; 5: e109.

26. Gu S, Kay MA. How do miRNAs mediate translational repression? Silence 2010; 1: 11

27. Huntzinger $E$, Izaurralde $E$. Gene silencing by microRNAs: contributions of translational repression and mRNA decay. Nat Rev Genet 2011; 12: 99-110.

28. Kole AJ, Swahari V, Hammond SM, Deshmukh M. miR-29b is activated during neuronal maturation and targets BH3-only genes to restrict apoptosis. Genes Dev 2011; 25: $125-130$

29. Zhou M, Liu Z, Zhao Y, Ding Y, Liu H, Xi Y et al. MicroRNA-125b confers the resistance of breast cancer cells to paclitaxel through suppression of pro-apoptotic $\mathrm{Bcl}-2$ antagonist killer 1 (Bak1) expression. J Biol Chem 2010; 285: 21496-21507.

30. Putcha GV, Deshmukh M, Johnson Jr EM. Inhibition of apoptotic signaling cascades causes loss of trophic factor dependence during neuronal maturation. J Cell Biol2000; 149 : 1011-1018.

31. Deshmukh M, Du C, Wang X, Johnson Jr EM. Exogenous smac induces competence and permits caspase activation in sympathetic neurons. J Neurosci 2002; 22: 8018-8027.

32. Putcha GV, Deshmukh M, Johnson Jr EM. BAX translocation is a critical event in neuronal apoptosis: regulation by neuroprotectants, BCL-2, and caspases. J Neurosci 1999; 19 : 7476-7485.

33. Potts PR, Singh S, Knezek M, Thompson CB, Deshmukh M. Critical function of endogenous XIAP in regulating caspase activation during sympathetic neuronal apoptosis. J Cell Biol 2003; 163: 789-799.

34. Wright KM, Linhoff MW, Potts PR, Deshmukh M. Decreased apoptosome activity with neuronal differentiation sets the threshold for strict IAP regulation of apoptosis. J Cell Biol 2004; 167: 303-313.

35. Wright KM, Smith MI, Farrag L, Deshmukh M. Chromatin modification of Apaf-1 restricts the apoptotic pathway in mature neurons. J Cell Biol 2007; 179: 825-832.

36. Yu LY, Jokitalo E, Sun YF, Mehlen P, Lindholm D, Saarma M et al. GDNF-deprived sympathetic neurons die via a novel nonmitochondrial pathway. J Cell Biol 2003; 163: 987-997.

37. Aalto AP, Sarin LP, van Dijk AA, Saarma M, Poranen MM, Arumae $U$ et al. Large-scale production of dsRNA and siRNA pools for RNA interference utilizing bacteriophage phi6 RNA-dependent RNA polymerase. RNA 2007; 13: 422-429.

38. Sun YF, Yu LY, Saarma M, Arumae U. Mutational analysis of N-Bak reveals different structural requirements for antiapoptotic activity in neurons and proapoptotic activity in nonneuronal cells. Mol Cell Neurosci 2003; 23: 134-143.

39. Bustin SA, Benes V, Garson JA, Hellemans J, Huggett J, Kubista M et al. The MIQE guidelines: minimum information for publication of quantitative real-time PCR experiments. Clin Chem 2009; 55: 611-622.

40. Schmittgen TD, Livak KJ. Analyzing real-time PCR data by the comparative $\mathrm{C}(\mathrm{T})$ method. Nat Protoc 2008; 3: 1101-1108.

Cell Death and Disease is an open-access journal published by Nature Publishing Group. This work is licensed under the Creative Commons Attribution-Noncommercial-No Derivative Works 3.0 Unported License. To view a copy of this license, visit http://creativecommons.org/licenses/by-nc-nd/3.0/ 